\title{
DATOS ABIERTOS ENLAZADOS (LOD) Y SU IMPLANTACIÓN EN BIBLIOTECAS: INICIATIVAS Y TECNOLOGÍAS
}

\author{
Linked open data (LOD) and its implementation in libraries: \\ Initiatives and technologies
}

Ana-Isabel Torre-Bastida, Marta González-Rodríguez y Esther VillarRodríguez

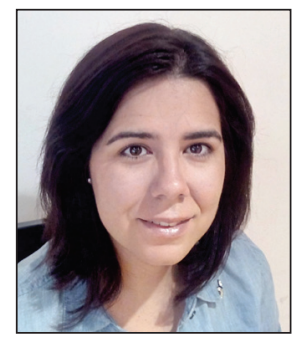

Ana-Isabel Torre-Bastida es ingeniera informática por la Universidad de Deusto y máster en sistemas informáticos avanzados por la Universidad del País Vasco. Doctorando, centra sus investigaciones en la web semántica, especialmente en datos abiertos enlazados. Es investigadora colaboradora en el centro tecnológico Tecnalia, donde lleva a cabo trabajos de aplicación de las tecnologías semánticas y de big data a varios sectores.

http://orcid.org/0000-0003-3005-1100

isabel.torre@tecnalia.com

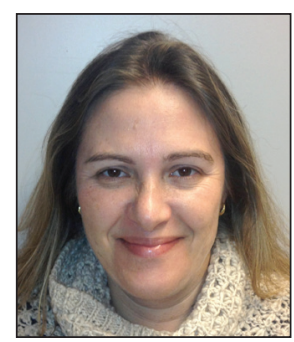

Marta González-Rodríguez, ingeniera informática por la Universidad del País Vasco y máster en tecnologías avanzadas de fabricación por la misma universidad, es gerente de Tecnologías Semánticas de Tecnalia. Participa en proyectos de I+D de ámbito nacional y europeo en el campo de las tecnologías semánticas e investiga en su aplicación a diferentes sectores. http://orcid.org/0000-0002-8785-8709

marta.gonzalez@tecnalia.com

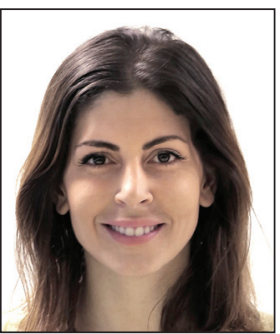

Esther Villar-Rodríguez es ingeniera informática por la Universidad de Deusto y máster en lenguajes y sistemas informáticos por la Universidad Nacional a Distancia (UNED). Doctorando e investigadora colaboradora en el centro tecnológico Tecnalia donde lleva a cabo investigaciones sobre procesamiento de lenguaje natural, analítica de datos y web semántica.

http://orcid.org/0000-0003-3343-3737

esther.villar@tecnalia.com

Unidad de Sistemas de Información e Interacción, División ICT-European Software Institute, Tecnalia Parque Tecnológico de Bizkaia Ibaizabal Bidea, Edif. 202. 48170 Zamudio, España

\section{Resumen}

Se analizan las principales iniciativas relacionadas con datos enlazados referidas a bibliotecas. La web de los datos se está convirtiendo en uno de los mayores repositorios globales de información, gracias a iniciativas como la de los datos abiertos enlazados (LOD o linked open data) bajo un único estándar. Su utilización ofrece grandes oportunidades a las bibliotecas, facilitando la publicación y gestión de sus datos mediante tecnologías semánticas y fomentando su conexión con otros repositorios, aumentando así su presencia y relevancia. Para asegurar el futuro de las bibliotecas en la web de los datos es necesario concienciar a los bibliotecarios de las oportunidades y los retos que plantean los LOD. Para ello y como referente, se presentan las principales iniciativas en este ámbito, junto con las organizaciones pioneras en la utilización de datos enlazados en el dominio de las bibliotecas.

\section{Palabras clave}

Web semántica; Datos abiertos enlazados; LOD; Conjuntos de datos; Datos abiertos; Bibliotecas; Catálogos digitales; Ontologías; RDF; Viaf; Europeana. 


\begin{abstract}
The web of data is becoming one of the largest global information repositories, thanks to initiatives like LOD (linked open data) that facilitate the standardized publication of open data. The use of this paradigm offers great opportunities for libraries, applying semantic technologies to expedite data management and publication and promoting their connection to other repositories, increasing their presence and impact. In order to ensure the future of libraries in the Web of data, it is necessary to raise awareness among librarians about LOD opportunities and challenges. With this aim, we present the major initiatives in this area, along with the pioneering organizations in the use of linked data in the library domain.
\end{abstract}

\title{
Keywords
}

Semantic web; Linked open data; LOD; Datasets; Open data; Libraries; Digital catalogs; Ontologies; RDF; VIAF; Europeana.

Torre-Bastida, Ana-Isabel; González-Rodríguez, Marta; Villar-Rodríguez, Esther (2015). Datos abiertos enlazados (LOD) y su implantación en bibliotecas: iniciativas y tecnologías". El profesional de la información, v. 24, n. 2, pp. 113-120.

http://dx.doi.org/10.3145/epi.2015.mar.04

\section{Introducción}

La Web se está convirtiendo en un enorme repositorio de información global compuesto por datos de diversos orígenes y dominios con sus interconexiones (Shiri, 2014). Esta web de los datos (Heath; Bizer, 2011) está alcanzado gran relevancia gracias al avance de la web semántica y sus tecnologías asociadas. La web semántica y en especial iniciativas como linked open data cloud (LODC) están alentando la publicación, interconexión y compartición de datos abiertos en la web, fomentando la interoperabilidad entre los distintos dominios.

Es importante destacar el potencial de la aplicación de estas técnicas en el área bibliotecaria: los $L O D$ pueden resolver gran parte de los retos asociados a la gestión de datos bibliotecarios.

En el sector de las bibliotecas se está llevando a cabo una importante labor de digitalización de contenidos (González; Bianchi; Vercelli, 2008), dirigiéndose hacia un nuevo concepto de bibliotecas digitales fomentado por redes como Europeana. http://www.europeana.eu

\section{La Web se está convirtiendo en un enor-} me repositorio de información compuesto por datos de diferentes orígenes y dominios así como sus interconexiones, lo que conocemos como web de los datos

Eric Miller (2004) indicó una serie de roles importantes a jugar por las bibliotecas en el campo de la web semántica:

- proveedores de colecciones de datos mediante tecnologías de la web semántica;

- responsables de adaptación de tesauros y servicios de las bibliotecas a la web de los datos enlazados;

- difusores de las lecciones aprendidas en este proceso de semantización de la información.

Su experiencia y conocimiento en el trabajo con vocabularios controlados las hace candidatas idóneas para migrar la información bibliotecaria al entorno de la web semántica. En el documento técnico final publicado por el World Wide
Web Consortium (W3C, 2011) se tratan en profundidad los beneficios que ofrecen los LOD y la situación actual del dominio bibliotecario, y se ofrecen recomendaciones para las bibliotecas que quieran acogerse a esta iniciativa. Ambos mundos se benefician de su mutua asociación, hecho que ya ha sido reconocido por gran número de instituciones del sector bibliotecario que están adoptando el paradigma LOD para la publicación de sus colecciones (Peset; Ferrer-Sapena; Subirats-Coll, 2011).

El objetivo de este artículo es analizar la implantación de los LOD en las bibliotecas, incluyendo los proyectos que hay detrás de los conjuntos de datos bibliográficos presentes en la nube de datos enlazados. Se presentan los retos que conlleva este proceso junto con posibles soluciones. Finalmente se extraerán una serie de conclusiones a partir de las cuales se pueden vislumbrar los próximos pasos de las bibliotecas en el ámbito de la web semántica y los datos libres enlazados.

\section{Linked open data en bibliotecas}

Los proyectos y recursos relacionados con la web semántica y los datos enlazados aplicados a bibliotecas han surgido de forma incesante durante los últimos años (Byrne; Goddard, 2010). Un ejemplo es el proyecto europeo LOD2, que promueve una mejora cualitativa de los conjuntos de datos de diversos dominios mediante tecnologías semánticas. Esto es debido principalmente a dos razones:

- la experiencia en vocabularios controlados, ontologías, metadatos y búsquedas convierte a los expertos del sector bibliotecario en candidatos perfectos para implementar los principios que promulga el paradigma linked data;

- la web semántica ofrece herramientas y estándares especialmente diseñados para publicación, compartición y enlazado de datos, que cubren los problemas con los que se han encontrado las bibliotecas a la hora de exponer sus datos y servicios a los usuarios en la web (bibliotecas y catálogos digitales). http://lod2.eu/WikiArticle/Project.html

A continuación presentamos una clasificación de los casos de aplicación de linked open data en instituciones (por parte de un conjunto de bibliotecas -Bibframe- o por casos particulares -Europeana, BNB...-), junto con conjuntos de datos que pueden ser de utilidad para el entorno bibliotecario. 


\subsection{Bibframe: nuevo modelo para expresar y conec- tar datos bibliotecarios}

En 2004 el W3C recomendó a las bibliotecas publicar sus almacenes de datos utilizando tecnologías de la web semántica, asegurándoles que así aumentaría su impacto digital y su utilidad social. Siguiendo sus indicaciones la Library of Congress (LC), creadora del estándar Marc21 utilizado como formato de catalogación y de intercambio de registros bibliotecarios, junto a otras bibliotecas y organizaciones usuarias de este formato digital (British Library, Deutsche Nationalbibliothek, George Washington University, National Library of Medicine, OCLC y Princeton University), lanzaron en mayo de 2011 el modelo Bibliographic framework transition (Bibframe) que fomenta la evolución del formato Marc21 hacia estándares basados en datos enlazados.

La Library of Congress es la primera biblioteca en aceptar la sustitución de Marc por datos enlazados, siendo la principal impulsora del nuevo modelo Bibframe

Bibframe constará de un área de demostración (datos de prueba), del vocabulario Bibframe y de las relaciones y correspondencias entre éste y los campos/subcampos de Marc21. La Library of Congress es la primera organización en aceptar la sustitución de Marc por los datos enlazados, siendo la principal impulsora de la iniciativa Bibframe. Existen numerosos trabajos sobre su futuro (Kroeger, 2013), y sobre cómo afectará a la catalogación (Fabeiro; Rovira-Jarque, 2013) o a la integración de las bibliotecas y sus catálogos en la Web actual (Svensson, 2013; Dean, 2013). http://bibframe.org

\subsection{Casos de aplicación directa en bibliotecas}

El objetivo de estas iniciativas es la creación de fuentes de datos de utilidad en el sector bibliotecario, siguiendo las pautas definidas por el paradigma linked open data. El creciente número de instituciones que se acogen a él permite establecer una estimación de la buena aceptación que está teniendo.

Las bibliotecas están realizando una importante labor de digitalización de contenidos dirigiéndose hacia un nuevo concepto de bibliotecas digitales fomentado por redes como Europeana

\section{Europeana linked open data}

Ha permitido que conjuntos de datos de portales europeos de patrimonio cultural (y en especial de bibliotecas) hayan sido transformados en datos enlazados y accesibles a través de data.europeana.eu, siguiendo el modelo EDM - Europeana data model, que tiene como fin estructurar y representar los datos con los que las instituciones de patrimonio cultural contribuyen a Europeana. Para ello sus creadores se centran en dos objetivos:

- mayor expresividad y flexibilidad que el actual modelo ESE (elementos semánticos de Europeana - Europeana semantic elements), al que reemplazará;

- seguir los principios fundamentales y buenas prácticas de la web semántica y linked data, a lo que Europeana quiere contribuir.

Para cumplir el segundo objetivo, el modelo se basa en estándares establecidos como RDF(s), OAI-ORE (open archive object reuse and exchange model), SKOS y Dublin core, y está disponible mediante una ontología OWL (Doerr et al., 2010).

\section{THE BRITISH NATIONAL BIBLIOGRAPHY}

as Linked Open Data

\begin{tabular}{l|c|c|c|c} 
Home & Getting Started & Documentation & FAQS & Contact Us \\
\hline
\end{tabular}

SPARQL editor

Enter your own SPARQL query below; alternatively click on one of the sample queries available. Furthe information about using the SPARQL editor is available here.

\section{- Sample queries}

o How do I search for a book with ISBN 9780729408745 ?

o How do I search for a serial title with ISSN 0955-6664?

- Find books on a particular subject, e.g crystallography

- Which titles by detective writer Ian Rankin appear in the BNB?

- List 50 authors born in 1945

o Find 50 books published in a particular place, e.g. York

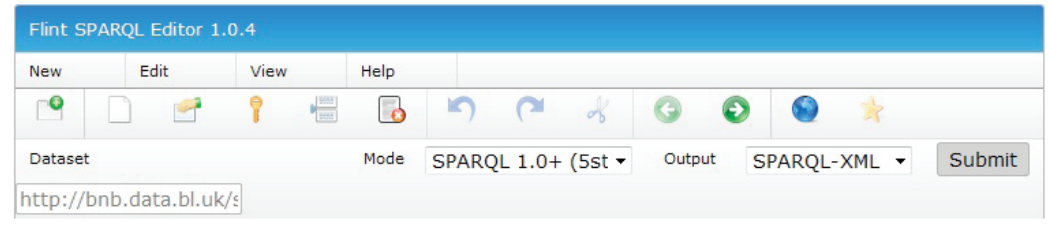

Figura 1. http://bnb.data.bl.uk
Actualmente están registrados en torno a 20 millones de objetos, descargables en formato RDF, o a través de consultas mediante un Sparql endpoint. Este no es más que una herramienta que permite realizar consultas Sparql sobre un grafo de entrada compuesto por tripletas RDF. A nivel técnico un Sparql endpoint implementa una interfaz descrita en la especificación Sparql protocol for RDF (Sprot) del $W 3 C$ que define una operación, un mensaje de entrada (compuesto por la consulta Sparql y el grafo sobre el cual ejecutarla) y dos mensajes 
de salida (uno para los resultados de la consulta y otro correspondiente a posibles errores causados por fallos en la sintaxis, semánticos, excepciones en tiempo de ejecución, u otros).

Existe numerosa bibliografía sobre el modelo de datos utilizado (Doerr et al., 2010) y la experiencia de implantación (Haslhofer; Isaac, 2011). En ella se explica la madurez del modelo EDM, el diseño de HTTP URI, y el rendimiento de repositorios RDF, así como los beneficios que tiene para otros proveedores definir sus datos mediante dicho modelo, ya que

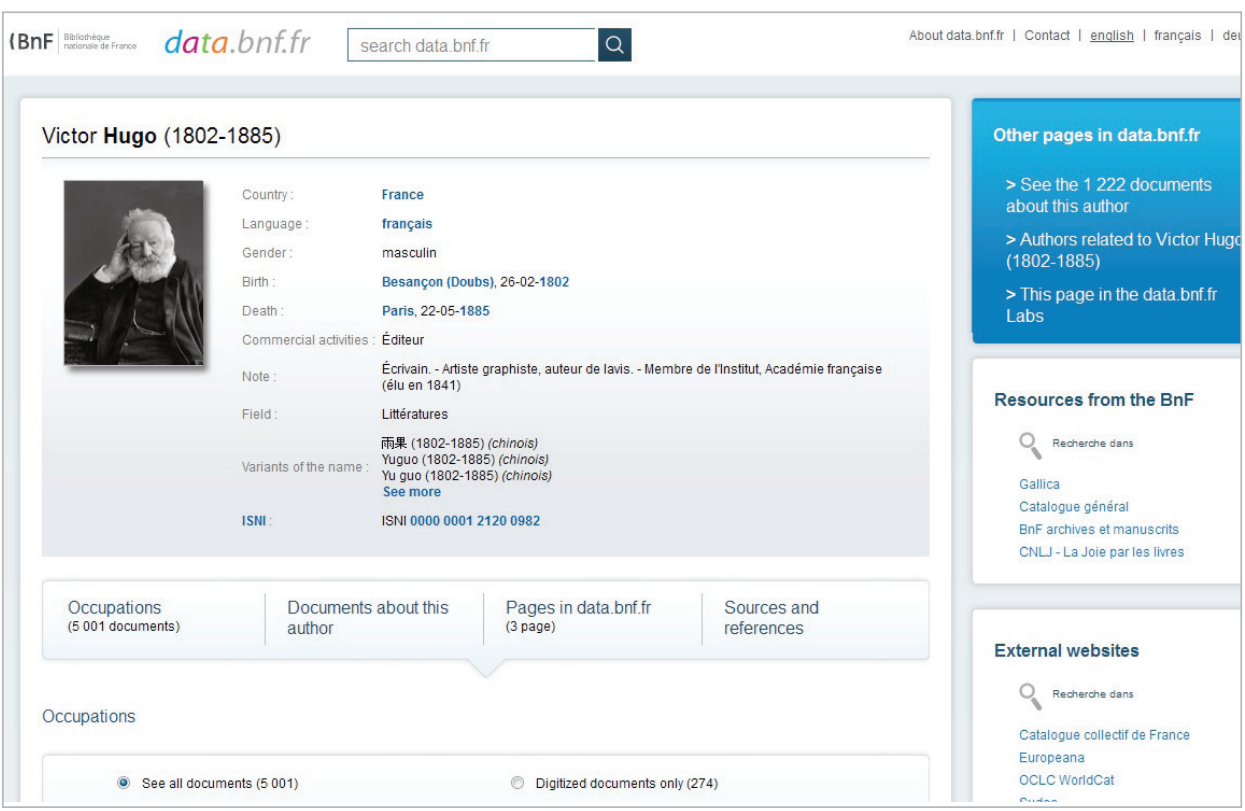

Figura 2. http://data.bnf.fr consiguen relacionarse con recursos semánticamente cercanos disponibles en la Web. Además Europeana ha aumentado su tamaño extrayendo información de la nube de LOD y enlazando sus recursos con los datos extraídos (Haslhofer et al., 2010).

http://www.europeana.eu

\section{British National Bibliography (BNB)}

La British Library mantiene la información de libros y revistas publicados o distribuidos en Irlanda y Reino Unido desde 1950. Actualmente estos registros están disponibles para ser descargados de forma gratuita en Marc21. Los datos enlazados de la BNB siguen dos modelos dependiendo de si los conceptos están referidos a libros o series: British Library data model for books y British Library data model for serials, respectivamente. La versión actual del conjunto de datos cuenta con 2,8 millones de descripciones de libros (93.583.853 tripletas)

La web semántica y linked open data cloud pueden ayudar a las bibliotecas a fomentar la publicación, interconexión y compartición de datos

La plataforma de datos enlazados que provee la BNB permite acceder al catálogo y las colecciones por varias interfaces: por Sparql endpoint, que permite al usuario el uso de un editor, o por búsqueda avanzada mediante texto plano (figura 1). Una descripción detallada de la labor realizada para implantar este paradigma y el modelo seguido se puede consultar en Deliot (2014).

http://bnb.data.bl.uk

\section{Bibliothèque nationale de France (BnF)}

El servicio de datos enlazados presentado por la Biblioteca nacional francesa se limita a publicar instancias de los autores y sus trabajos, un total de 1.600 autores y 4.000 trabajos. Estos datos pueden consultarse mediante una herramienta de búsqueda asistida. En la figura 3 puede apreciarse como

se compone de listas desplegables y listados de conceptos (autores, temáticas y obras). Para más detalle se puede consultar el caso de uso presentado al W3C o el trabajo de Simon et al. (2013), donde se muestran los beneficios obtenidos por la biblioteca al haber publicado sus registros en la web de datos.

\section{Biblioteca Nacional de España (BNE)}

El conjunto de datos datos.bne.es ofrece el acceso al catálogo bibliográfico y de autoridades de la Biblioteca Nacional de España (BNE) como datos enlazados. Los registros en Marc21 han sido transformados a RDF y modelados utilizando principalmente las ontologías de la IFLA (International Federation of Library Associations and Institutions). El proceso (Vila-Suero; Villazón-Terrazas; Gómez-Pérez, 2012) se ha automatizado en parte mediante el programa Marimba, que permite la transformación automática a RDF y el descubrimiento de enlaces hacia otros conjuntos de datos, como DBpedia o Viaf. Para esta segunda tarea también se han ayudado de Silk. Para la publicación y consulta se ha utilizado el repositorio RDF Virtuoso (el Sparql endpoint puede verse en la figura 4) y la interface para Sparql endpoint pubby.

Cuenta con 7 millones de registros bibliográficos y de autoridades. Para la definición de los registros se han utilizado propiedades extraídas principalmente de ontologías diseñadas por la IFLA, como:

- requerimientos funcionales para registros bibliográficos (FRBR);

- requisitos funcionales para datos de autoridad de materia (FRSAD);

- descripción bibliográfica internacional normalizada (ISBD),

y otras como:

- Resource description and access (RDA) y

- Dublin core metadata element set.

Los datos están publicados de acuerdo con las especificaciones y condiciones de la licencia Creative Commons Zero (CCO). 


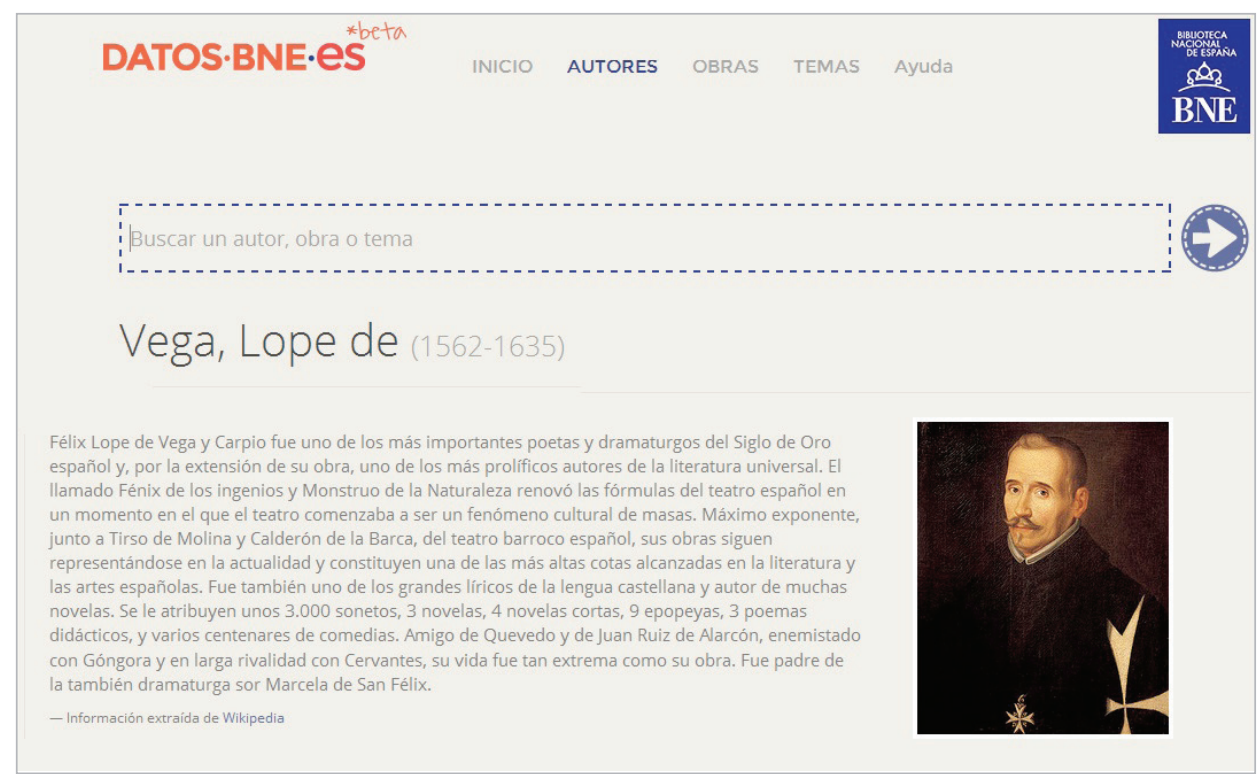

Figura 3. http://datos.bne.es

http://www.bne.es/es/Inicio/Perfiles/Bibliotecarios/ DatosEnlazados/index.html

\section{Library of Congress subject headings (LCSH)}

Es el catálogo de materias de la Library of Congress de los EUA desde 1898, siendo utilizado por múltiples instituciones a nivel internacional para el acceso clasificado a colecciones por materia. El tamaño de este conjunto de datos es de 7.332.816 tripletas RDF. Los vocabularios utilizados para su definición son Metadata authority description schema (MADS) y Simple knowledge organization system (SKOS) (Summers et al., 2008). Este conjunto de datos únicamente se encuentra conectado a los datos del conjunto Rameau subject headings (Semantic interoperability to access cultural heritage, Stitch) mantenido por la Bibliothèque nationale de France.

http://www.w3.org/2004/02/skos

\subsection{Fuentes de datos abiertas y enlazadas de utilidad en el ámbito bibliotecario}

A continuación se citan conjuntos de datos abiertos de linked open data cloud, que son de utilidad para cualquier institución que quiera publicar sus datos siguiendo el paradigma LOD. Algunos de estos conjuntos pueden ser un referente a la hora de enlazar recursos (DBPedia, Viaf) y otros permiten encontrar información útil y reutilizable durante la catalogación (Lobid, Open library...).

\section{DBpedia}

Es un proyecto para estructurar la información contenida en la Wikipedia y hacerla accesible en la Web. Está interconectado con GeoNames, Musicbrainz, CIA world factbook, Proyecto Gutenberg y Eurostat entre otros. Su base de conocimiento en la versión en inglés describe más de 4 millones de conceptos, clasificados mediante una ontología. Uno de sus principales atractivos es que se ha traducido a 119 idiomas; si se tienen en cuenta todas las versiones llega a definir 24,9 millones de conceptos. Podemos hacer consultas al repositorio de tripletas a través de su Sparql endpoint. Tiene 2.460 millones de tripletas RDF, de las cuales 45 millones son enlaces a otras fuentes de datos. La ontología que utiliza DBpedia, implementada en OWL, es poco profunda, cubre un dominio transversal $y$ ha sido creada manualmente basándose en conceptos extraídos de Wikipedia. La ontología cubre 529 clases que forman una jerarquía de subsunción y se describen por 2.333 propiedades diferentes. Muchos trabajos se nutren de la información contenida en este repositorio y su naturaleza transversal, para mejorar sus contenidos. En el dominio en estudio DBpedia puede suponer una fuente de información a agregar a la información estructura extraída de las bibliotecas digitales (Chao; Zhang; Xing, 2011). http://dbpedia.org/About

\section{Viaf (Virtual international authority file)}

Combina varios archivos de autoridad de nombres en un solo servicio hospedado en OCLC (Online Computer Library Center, Dublin, Ohio, EUA). Se trata de una gran base de datos de personas y organizaciones que se encuentran definidas en los catálogos de las bibliotecas. La estructura canónica de un uri Viaf es:

http://viaf.org/viaf/[valor numérico]

Ejemplo: $h$ ttp://viaf.org/viaf/49224511

Numerosas bibliotecas utilizan este servicio como registro de autoridad, un ejemplo es la Biblioteca Nacional de Alemania (Bennett et al., 2007).

\section{Lobid. Index of libraries and related organizations}

Contiene un directorio de bibliotecas y organizaciones relacionadas, que sigue los principios de los datos enlazados. La mayoría de los datos provienen del Online-ISIL - Directory y de la MARC Organization Codes Database. El número de instituciones identificadas supera las 4.000. Los uris están basados en el estándar internacional ISIL (International standard identifier for libraries and related organizations).

Este repositorio puede ser utilizado por medio de un API, lo que ha permitido su integración en aplicaciones y servicios web de utilidad para el ciudadano (Hausenblas, 2011), como fuente o directorio de consulta sobre bibliotecas cercanas a éste. Además, como directorio de bibliotecas está enlazado con fuentes de datos como DBpedia y OpenLibrary. http://lobid.org/api

\section{Open library}

Es una base de datos de libros, colaborativa y de acceso público. Identifica y publica una página web con la información de cada libro editado. En enero de 2015 han recogido cerca de 30 millones de registros (14 millones están ya disponibles 
en el sitio). Se puede buscar entre millones de registros de libros, refinar los resultados por criterios, y buscar a texto completo en 230.000 libros digitalizados. Esta versión tiene la característica de estar enlazada con otras fuentes de LOD como DBpedia, LinkedLCCN o Marc codes.

El principal obstáculo en la implantación de este tipo de iniciativas es la falta de concienciación de sus beneficios por parte de los bibliotecarios

\section{Retos a superar por la comunidad bibliotecaria}

En primer lugar hay que resaltar las posibilidades que ofrece la utilización de un único formato común para todos los datos (RDF) en cuanto a interoperabilidad e integración con sistemas y datos propios o externos, evitando el caos actual en la mezcla de sistemas y formatos en las bibliotecas digitales.

Modelos como los mencionados anteriormente (BibFrame, Europeana) o RDA (Recursos, descripción y acceso) de instrucciones para descripción y acceso de los recursos bibliotecarios (Coyle; Hillmann, 2007; Tillett, 2011), intentan facilitar esta tarea.

En segundo lugar, las tecnologías semánticas pueden ayudar en el desarrollo de mecanismos de búsqueda más potentes, que cubran mejor las expectativas del usuario, posibilitando entre otras funciones:

- consultas más específicas: gracias a editores o constructores de consultas Sparql, como Autosparql (Lehmann; Bühmann, 2011), SINA (mecanismo de búsqueda sobre datos) o SWIP (Pradel et al., 2013), o a exploradores de datos como rkbexplorer o snorql:

http://sina.aksw.org

- rankings por relevancia: existen numerosos trabajos que estudian la generalización o aproximación de la consulta del usuario y ordenan las preguntas en función de su similitud (Hurtado; Poulovassilis; Wood, 2009; Huang; Liu; Zhou, 2010; Poulovassilis; Wood, 2010). Esto puede ser muy útil en el dominio de las bibliotecas para usuarios inexpertos en el uso de sus catálogos que no estén seguros de su pregunta y que con estas técnicas puedan conseguir una mayor exhaustividad (recall) en los resultados.

- la federación de distintas fuentes de datos es un área de estudio ampliamente investigada y con soluciones muy potentes como FedX (Schwarte et al., 2011), Yars2 (Harth et al., 2007) o DARQ3 (Quilitz; Leser, 2008). Su uso permite abstraerse de las dificultades técnicas de consultar uno a uno los conjuntos de datos de la Web y considerarla como un único repositorio global.

Problemas que llevan años afectando a las bibliotecas como los relacionados con el control de autoridad (Viaf es un servicio que puede dar solución a este problema), o el proceso de catalogación, se pueden solucionar con el uso de tecnologías semánticas. La catalogación es un proceso pesado y costoso que podría verse simplificado si se estandariza y comparte la información de catalogaciones previas pudiendo así ser explotada por la comunidad bibliotecaria en general. Este es uno de los objetivos del proyecto europeo Aliada.

http://www.aliada-project.eu

Se pueden destacar dos problemas específicos que surgen durante la implantación de los linked open data en el área bibliotecaria:

- retos tecnológicos a los que se deben enfrentar los bibliotecarios al intentar implantar este tipo de iniciativas. Aunque los profesionales de bibliotecas se encuentren familiarizados con los lenguajes de definición o los vocabularios controlados, no lo suelen estar con las tecnologías semánticas (Heath; Bizer, 2011; Corcho; FernándezLópez; Gómez-Pérez, 2006) y no existen herramientas adaptadas y eficientes que les faciliten tareas como la catalogación o la publicación de los datos;

- falta de concienciación de los beneficios que proporcionaría su implantación. Esto representa un grave problema ya que los beneficios de los datos enlazados se aprecian mejor y crecen al aumentar el número de datos enlazados disponibles. Sin un alto volumen de información expuesta e interconexiones entre dicha información, los datos enlazados no son más que un mero estándar para la definición de metadatos, lo que para los bibliotecarios no deja de ser uno más entre tantos otros. Por lo tanto y para que esta aproximación pueda llegar a ser una realidad, es de vital importancia dar a conocer las iniciativas actuales y fomentar el seguimiento de su ejemplo.

Un reto para los bibliotecarios es la consolidación de la posición de los conjuntos de datos del dominio de las bibliotecas de forma que puedan ser utilizados como fuente de conocimiento por la propia comunidad

\section{Conclusiones}

Se ha intentado destacar que los datos enlazados representan una gran oportunidad para las bibliotecas, ya que les permiten integrar sus recursos con la inmensa fuente de información que es la Web, mejorando y en algunos casos posibilitando la exposición y visibilidad de los catálogos de sus colecciones.

Las bibliotecas pueden utilizar las tecnologías semánticas para mejorar la gestión de las tareas de publicación, compartición, consulta o persistencia, permitiendo cubrir de una forma más eficiente y completa las expectativas de interacción que tiene el usuario hacia las nuevas bibliotecas y catálogos digitales. También permiten mejorar tareas típicas de la biblioteconomía como la catalogación mediante la reutilización de catalogaciones previas almacenadas en otros conjuntos de datos (proyecto Aliada). Teniendo todo esto en cuenta, junto con la existencia de proyectos como 
Bibframe o Europeana y el listado de organizaciones que ya han acogido la iniciativa LOD, en nuestra opinión está claro que el futuro de las bibliotecas pasa por acogerse al paradigma LOD.

Ante esto queremos resaltar las dos posibles líneas de trabajo futuro que consideramos más prometedoras:

- promulgar en la comunidad este tipo de iniciativas para conseguir un mayor número de colecciones expuestas, que puedan ser enlazadas creando una gran colección global accesible. Es decir, generar una web de los datos específica del dominio de las bibliotecas que pueda ser utilizada como fuente de conocimiento y como referencia para otros dominios;

- crear servicios de valor para el usuario utilizando tecnologías semánticas (basándose en herramientas ya existentes y citadas anteriormente); por ejemplo, nuevos sistemas semánticos de búsqueda conociendo el contexto del usuario y federados entre los diferentes repositorios que le interesen.

\section{Bibliografía}

Bennett, Rick; Hengel-Dittrich, Christina; O’Neill, Edward T.; Tillett, Barbara B. (2006). "Viaf (virtual international authority file): Linking die Deutsche Bibliothek and Library of Congress name authority files". International cataloguing and bibliographic control, v. 36, n. 1, pp. 12-18.

Also: In: World library and information congress: $72^{\text {nd }}$ IFLA general conf and council, 20-24 August 2006, Seoul, Korea. http://archive.ifla.org/IV/ifla72/papers/123-Bennett-en.pdf

Byrne, Gillian; Goddard, Lisa (2010). "The strongest link: Libraries and linked data". D-Lib magazine, v.16, pp.11/12.

http://research.library.mun.ca/3/2/emtac/10_ goddardbyrne final.pdf

http://dx.doi.org/10.1045/november2010-byrne

Chao, Lemen; Zhang, Yong; Xing, Chunxiao (2011). "Improving query suggestion for digital libraries". En: Computer software and applications conf workshops (Compsacw), 2011 IEEE 35 th Annual, pp. 428-434.

http://dx.doi.org/10.1109/COMPSACW.2011.78

Corcho, Óscar; Fernández-López, Mariano; Gómez-Pérez, Asunción (2006). "Ontological engineering: principles, methods, tools and languages". Ontologies for software engineering and software technology, pp. 1-48.

http://oa.upm.es/5457

http://dx.doi.org/10.1007/3-540-34518-3_1

Coyle, Karen; Hillmann, Diane (2007). "Resource description and access (RDA): Cataloging rules for the $20^{\text {th }}$ century". D-Lib magazine, n. 13, v. 1, pp. 3.

http://www.dlib.org/dlib/january07/coyle/01coyle.html http://dx.doi.org/10.1045/january2007-coyle

Dean, Jason W. (2013). "Charles A. Cutter and Edward Tufte: coming to a library near you, via Bibframe". The library with the lead pipe, 4 December.

http://www.inthelibrarywiththeleadpipe.org/2013/charlesa-cutter-and-edward-tufte-coming-to-a-library-near-youvia-bibframe
Deliot, Corine (2014). Publishing the British National Bibliography as linked open data. The British Library.

http://www.bl.uk/bibliographic/pdfs/publishing_bnb_as lod.pdf

Doerr, Martin; Gradmann, Stefan; Hennicke, Steffen; Isaac, Antoine; Meghini, Carlo; Van-de-Sompel, Herbert (2010). "The Europeana data model (edm)". En: World library and information congress: $76^{\text {th }}$ IFLA general conference and as sembly, pp. 10-15.

http://conference.ifla.org/past-wlic/2010/149-doerr-en.pdf

Fabeiro, Rosa; Rovira-Jarque, Anna (2013). "Linked Open Data, el futur de la catalogació". En: Coneixement i información en obert. Cobdc, 21 nov.

http://hdl.handle.net/2445/47997

Gónzalez, Marta; Bianchi, Stefano; Vercelli, Gianni (2008). "Semantic framework for complex knowledge domains". En: Intl semantic web conf (posters \& demos).

http://wifo5-03.informatik.uni-mannheim.de/bizer/pub/ iswc2008pd/iswc2008pd_submission_17.pdf

Harth, Andreas; Umbrich, Jürgen; Hogan, Aidan; Decker, Stefan (2007). "Yars2: A federated repository for querying graph structured data from the web". En: ISWC'07/ ASWC'07, pp. 211-224.

http://dx.doi.org/10.1007/978-3-540-76298-0_16

Haslhofer, Bernhard; Isaac, Antoine (2011). "data.europeana.eu: The Europeana linked open data pilot". En: Int/ conf on Dublin core and metadata applications, pp. 94-104.

http://www.few.vu.nl/ aisaac/papers/dataeuropanea_ submitted.pdf

Haslhofer, Bernhard; Momeni, Elaheh; Gay, Manuel; Simon, Rainer (2010). "Augmenting Europeana content with linked data resources". En: Procs of the $6^{\text {th }}$ Intl conf on semantic systems, pp. 40, ACM.

http://dx.doi.org/10.1145/1839707.1839757

Hausenblas, Michael (2011). "Utilizing linked open data in applications". En: Procs of the Intl conf on web intelligence, mining and semantics.

http://doi.acm.org/10.1145/1988688.1988697

Heath, Tom; Bizer, Christian (2011). “Linked data: Evolving the web into a global data space". Synthesis lectures on the semantic web: theory and technology, v. 1, n. 1, pp. 1-136. http://linkeddatabook.com/editions/1.0 http://dx.doi.org/10.2200/S00334ED1V01Y201102WBE001

Huang, Hai; Liu, Chengfei; Zhou, Xiaofang (2012). "Approximating query answering on RDF databases". World wide web, v. 15, n. 1, pp. 89-114.

http://dx.doi.org/10.1007/s11280-011-0131-7

Hurtado, Carlos A.; Poulovassilis, Alexandra; Wood, Peter T. (2009). "Ranking approximate answers to semantic web queries". The semantic web: Research and applications. Lecture notes in computer science, v. 5554, pp. 263-277. http://dx.doi.org/10.1007/978-3-642-02121-3_22

Kroeger, Angela (2013). "The road to Bibframe: the evolution of the idea of bibliographic transition into a post-MARC future". 
Cataloging \& classification quarterly, v. 51, n. 8, pp. 873-890. http://dx.doi.org/10.1080/01639374.2013.823584

Lehmann, Jens; Bühmann, Lorenz (2011). “AutoSparql: Let users query your knowledge base". The semantic web: Research and applications. Lecture notes in computer science, v. 6643, pp. 63-79.

http://dx.doi.org/10.1007/978-3-642-21034-1_5

Miller, Eric (2004). Digital libraries and semantic web. W3C. http://www.w3.org/2001/09/06-ecdl/Overview.html

Peset, Fernanda; Ferrer-Sapena, Antonia; Subirats-Coll, Imma (2011). "Open data y linked open data: su impacto en el área de bibliotecas y documentación". El profesional de la información, v. 20, n. 2, pp. 165-174.

http://eprints.rclis.org/21003/1/06.pdf

http://dx.doi.org/10.3145/epi.2011.mar.06

Poulovassilis, Alexandra; Wood, Peter T. (2010). “Combining approximation and relaxation in semantic web path queries". The semantic web, ISWC 2010. Lecture notes in computer science, v. 6496, pp. 631-646.

http://dx.doi.org/10.1007/978-3-642-17746-0_40

Pradel, Camille; Peyet, Guillaume; Haemmerlé, Ollivier; Hernandez, Nathalie (2013). "Swip at qald-3: results, criticisms and lesson learned". En: Procs of the question answering over linked data lab (QALD-3) at CLEF.

http://ceur-ws.org/Vol-1179/CLEF2013wn-QALD3PradelEt2013.pdf

Quilitz, Bastian; Leser, Ulf (2008). "Querying distributed RDF data sources with Sparql". En: ESWC'08 Procs of the $5^{\text {th }}$ European semantic web conf on the semantic web: research and application, pp. 524-538.

http://zope.informatik.hu-berlin.de/forschung/gebiete/ wbi/research/publications/2008/DARQ-FINAL.pdf

Schwarte, Andreas; Haase, Peter; Hose, Katja; Schenkel, Ralf; Schmidt, Michael (2011). "FedX: a federation layer for distributed query processing on linked open data". En: The semantic web: research and applications. Lecture notes in computer science, v. 6644, pp. 481-486.

http://www2.informatik.uni-freiburg.de/ mschmidt/docs/ iswc11_fedx.pdf

http://dx.doi.org/10.1007/978-3-642-21064-8_39

Shiri, Ali (2014). "Linked data meets big data: A knowledge organization systems perspective". En: Advances in classification research online, v. 24, n. 1.

https://journals.lib.washington.edu/index.php/acro/ article/viewFile/14672/12312

http://dx.doi.org/10.7152/acro.v24i1.14672

Simon, Agnès; Wenz, Romain; Michel, Vincent; Di-Mascio, Adrien (2013). "Publishing bibliographic records on the web of data: opportunities for the BnF (French National Library)". En: The semantic web: Semantics and big data, pp. 563-577. http://eswc-conferences.org/sites/default/files/ papers2013/simon.pdf

Summers, Ed; Isaac, Antoine; Redding, Clay; Krech, Dan (2008). "LCSH, SKOS and linked data". En: Procs of the IntI conf on Dublin core and metadata applications (DC 2008), Berlin, Germany, Sept. 22-26.

Svensson, Lars G. (2013). "Are current bibliographic models suitable for integration with the web?". Information standards quarterly, Winter, v. 25, n. 4, pp. 6-13. http://dx.doi.org/10.3789/isqv25no4.2013.02

Tillett, Barbara B. (2011). "Keeping libraries relevant in the semantic web with resource description and access (RDA)". Serials: The journal for the serials community, v. 24, n. 3, pp. 266-272.

http://dx.doi.org/10.1629/24266

Vila-Suero, Daniel; Villazón-Terrazas, Boris; Gómez-Pérez, Asunción (2013). "datos.bne.es: A library linked dataset". Semantic web, v. 4, n. 3, pp. 307-313.

http://dx.doi.org/10.3233/SW-120094

W3C (2011). Library Linked Data Incubator Group, final report. http://www.w3.org/2005/Incubator/IId/XGR-Ild-20111025

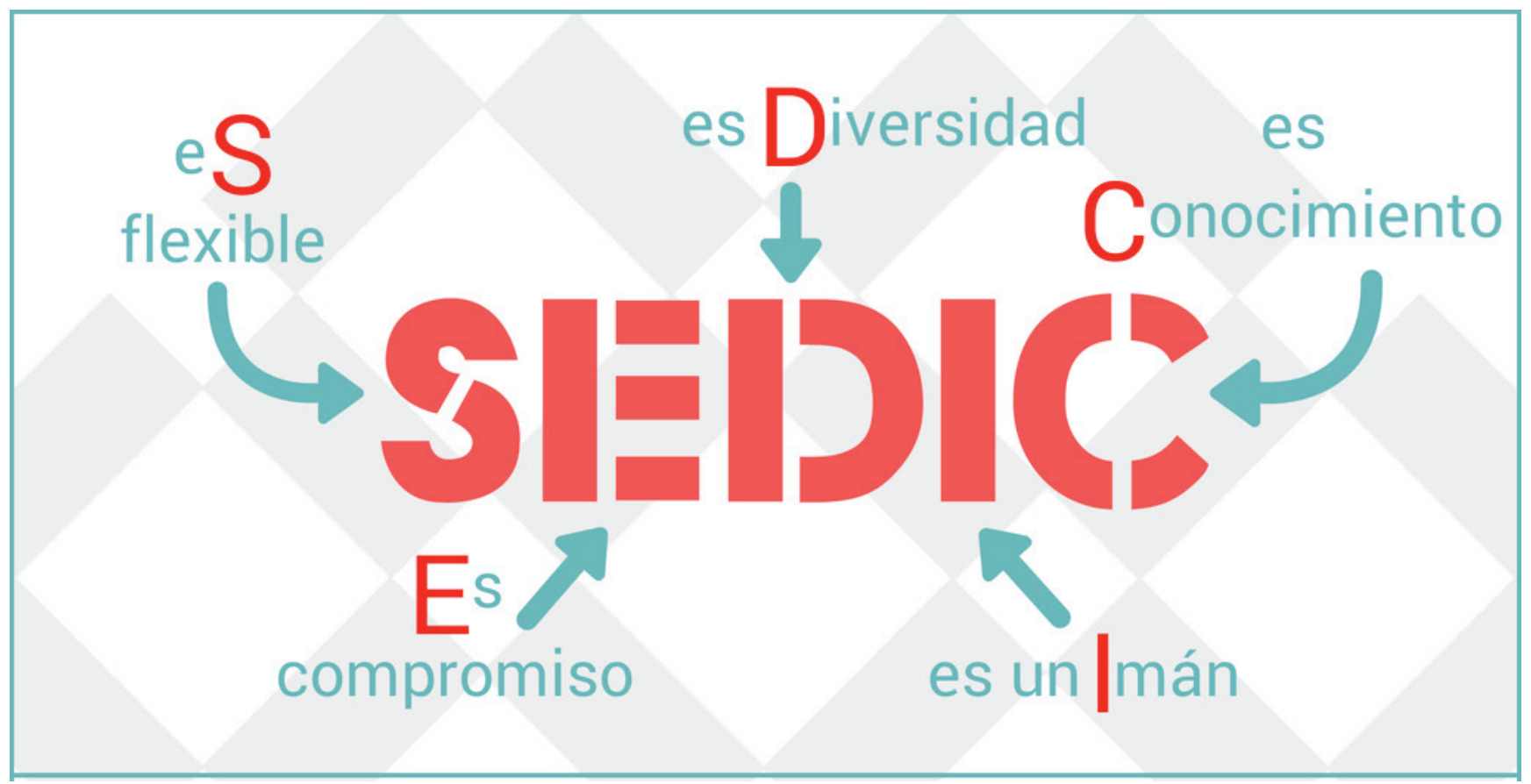

